\title{
On the Problem of Finding "Sets Ensuring Linearly Independent Transversals" (SELIT), and its Application to Network Coding
}

\author{
Hirah Malik, Cedric Adjih \\ Inria, Saclay \\ 91120 Palaiseau, France \\ $<$ name $>.<$ surname $>@$ inria.fr
}

\author{
Michel Kieffer \\ Université Paris-Saclay, CNRS \\ CentraleSupelec, L2S \\ 91192 Gif-sur-Yvette, France \\ michel.kieffer@12s.centralesupelec.fr
}

\author{
Claudio Weidmann \\ ETIS UMR8051, CY University, \\ ENSEA, CNRS \\ 95000 Cergy, France \\ claudio.weidmann@ensea.fr
}

\begin{abstract}
This paper introduces a new formal mathematical problem initially motivated by an application of Network Coding (NC) to Information Centric Networks (ICN). It is of more limited scope but is remotely inspired by the well-known index coding problem. It is presented as follows: "given a vector space, can one construct several subsets of vectors, such that when drawing arbitrarily one vector from each subset, the selected vectors would be always linearly independent?'. Answering this question is a step to construct an ICN efficient scheme with NC. We prove that our previously introduced construction is the only possible solution for a large family of constructions. This is an important result by itself. It also implies that any alternate solutions are outside this family and we propose one example.
\end{abstract}

\section{INTRODUCTION}

The Efficient delivery of source content from some servers to one or several clients is one of the common network operations. In this paper, we are motivated by the use of Information Centric Networking [1] for such content delivery. IP protocol is not used; instead, clients send Interest packets to the network (not to a specific server); the Interests find their way to one or several servers with content; the content is then returned in Data packets on the reverse path. Additionally, content can be cached inside the network to potentially satisfy future requests.

Our starting point is the inefficiency illustrated by the scenario in Fig. 1, with basic ICN. Given a client and two servers, the client sends one request (Interest) labeled $I_{1}$ to the network to retrieve one unit of source content. Notice that the Interest $I_{1}$ is forwarded on two paths. The servers $S_{1}$ and $S_{2}$ reply with content $Q_{1}=Q_{1}^{\prime}=P_{1}$. Nevertheless, only the first Data reply $Q_{1}$ is useful to the client, and $Q_{1}^{\prime}$ is redundant.

Inefficiency occurs because 1) Interest $I_{1}$ is forwarded on more than one path and 2) because the Data packets $Q_{1}$ and $Q_{1}^{\prime}$ carry identical content. The rationale for forwarding Interest $I_{1}$ on multiple paths is a potential throughput increase. The

This research was partly supported by Labex DigiCosme (project ANR11LABEX0045DIGICOSME) operated by ANR as part of the program "Investissement d'Avenir" Idex ParisSaclay (ANR11IDEX000302)

(C) IFIP 2020. This is the author's version of the work. It is posted here by permission of IFIP for your personal use. Not for redistribution. The definitive version was published in the Proceedings of PEMWN 2020

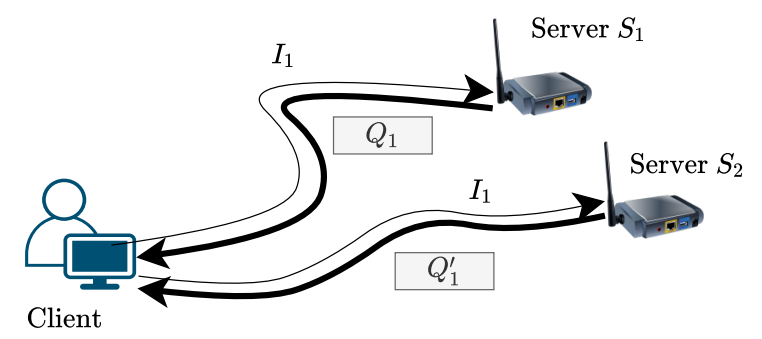

Fig. 1. Can ICN make efficient use of multiple paths?

real issue is that $Q_{1}$ is equal to $Q_{1}^{\prime}: Q_{1}^{\prime}$ might have returned more useful information.

One interesting solution is to adopt network coding(NC) [2], to improve the performance of ICN, as proposed in [3]. There is currently ongoing work in standardization on combining NC and ICN [4]. (Linear) network coding consists of viewing all packets as vectors of elements of a finite field and performing algebraic operations on them, such as linear combinations.

In our case, if the replies $Q_{1}$ and $Q_{1}^{\prime}$ were coded packets, it could be possible for the servers to return, for example, $Q_{1}=$ $P_{1}+2 P_{2}$ and $Q_{1}^{\prime}=P_{1}+P_{2}$. Then $Q_{1}$ and $Q_{1}^{\prime}$ would no longer be redundant for the client, would be able to recover source contents $P_{1}$ and $P_{2}$, effectively doubling the throughput.

However, this is only half of the solution. Assuming the client targets high throughput and simultaneously sends multiple parallel Interests as in Fig. 2. Each of them would

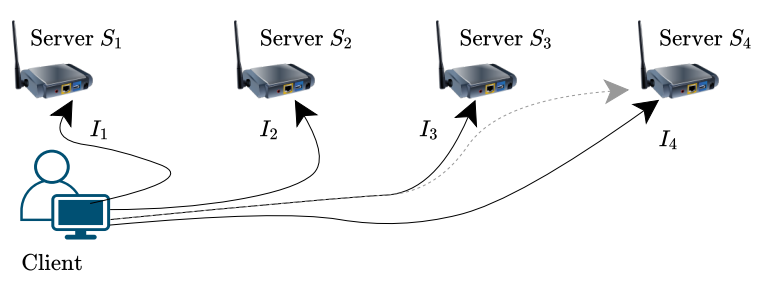

Fig. 2. What should the servers reply to each Interest sent in parallel? 
ideally bring back innovative content, but the question is how to guarantee this. To address this problem, in [5], [6], we proposed a construction called MILIC allowing one to build Multiple Interests for Linearly Independent Content. Motivated by the search for alternate solutions with potentially even better performance, we looked for generalizations of this construction. We could prove that a large class of generalizations are equivalent (isomorphic) to MILIC. The main contribution of this article is the proof for this fact in Theorem 4; this is of high interest because it limits the search space for alternate constructions, and the steps of the proof themselves shed light on properties the alternate solutions must have (or not have). Then, we indeed exhibit one different family of solutions.

The article is organized as follows: notations are introduced in Sect. II; a mathematical formulation of the problem is given in Sect. III; Sect. IV discusses its solutions, including MILIC; in Sect. V, a generic family of constructions (for potential solutions), is defined; Sect. VI and Sect. VII contains the proof that solutions from this family are included in MILIC; Sect. VIII shows alternative constructions; Sect. IX concludes.

\section{PREliminary Notations AND DeFinitions}

Let $\llbracket k \rrbracket \stackrel{\text { def }}{=}\{1,2, \ldots k\}$ be the set of integers from 1 to $k$. $F$ denotes a finite field with $|F|>2, F^{*} \stackrel{\text { def }}{=} F \backslash\{0\}$, and $F^{n}$ is the vector space of dimension $n \geqslant 1$ over $F$

$$
F^{n}=\left\{\left(\begin{array}{llll}
x_{1} & x_{2} & \ldots & x_{n}
\end{array}\right) \mid x_{1} \in F, x_{2} \in F, \ldots, x_{n} \in F\right\} .
$$

The vector $e_{i}=\left(\begin{array}{lll}0 \cdots 0 & 1 & 0 \cdots 0\end{array}\right)$ is the $i$-th canonical vector of $F^{n}$. $\underbrace{0 \cdots \cdot}_{i-1 \text { zeros }}$

Definition 1 (Encoding vector of a linear combination). If a coded packet $Q$ is a linear combination of the source content $P_{1}, P_{2}, \ldots P_{n}$, with $Q=\alpha_{1} P_{1}+\alpha_{2} P_{2}+\ldots+\alpha_{n} P_{n}$, with $\forall i \in \llbracket n \rrbracket, \alpha_{i} \in F$, then the encoding vector of $Q$ is $v=$ $\left(\begin{array}{llll}\alpha_{1} & \alpha_{2} & \ldots & \alpha_{n}\end{array}\right)$.

Definition 2 (Transversal). Let $E$ be an arbitrary set. Consider a family of $k$ subsets of $E$, denoted $\mathcal{A}=\left(\mathcal{A}_{1}, \ldots, \mathcal{A}_{k}\right)$. $A$ transversal $T$ of $\mathcal{A}$ is a set obtained by picking one element in each subset $\mathcal{A}_{i}: T=\left\{a_{1}, a_{2}, \ldots a_{k}\right\}$ with $a_{i} \in \mathcal{A}_{i}, \forall i \in \llbracket k \rrbracket$.

\section{Multipath Retrieval and The Problem of Sets ENSURING LINEARLY INDEPENDENT TRANSVERSALS}

Consider the content retrieval problem in the context of ICN illustrated in Fig. 2. A client wants to retrieve $n$ segments $P_{1}, P_{2}, \ldots, P_{n}$ of source content. The client sends $n$ Interest packets $\left(I_{i}\right)_{i=1 \ldots n}$. The reply to the $i$-th interest $I_{i}$ from any server will be a linear combination whose encoding vector comes from a predefined set $\mathcal{A}_{i}$. The problem considered in this paper is to build a good family of $n$ sets $\left(\mathcal{A}_{i}\right)_{i \in \llbracket n \rrbracket}$. Such good family has to satisfy the following properties.

- Completeness (Fig. 2): When $n$ coded packets with encoding vectors $v_{1} \in \mathcal{A}_{1}, v_{2} \in \mathcal{A}_{2}, \ldots v_{n} \in \mathcal{A}_{n}$ are received by the client, it should be able to recover $P_{1}, P_{2} \ldots P_{n}$.

- High diversity (Fig. 1): If $k$ coded packets with encoding vectors $v_{1} \ldots v_{k}$ are received, some potentially drawn from the same sets (e.g., the following is possible: $v_{1} \in \mathcal{A}_{7}, v_{2} \in$
$\left.\mathcal{A}_{7}, v_{3} \in \mathcal{A}_{5}, v_{4} \in \mathcal{A}_{5} \ldots\right)$, they should provide nonredundant information with high probability.

Our construction, MILIC (see Definition 3 in what follows), introduced in [5], satisfies both properties. In this work, because high diversity is arguably more difficult to formulate precisely for being inherently probabilistic, we ignore this aspect ${ }^{1}$. Instead, we focus on ensuring the completeness property. This can be recast as finding Sets Ensuring Linearly Independent Transversals (SELIT) ${ }^{2}$ such as:

Problem 1 (SELIT problem). Given a finite field $F$, and the vector space $F^{n}$ of dimension $n$ over $F$ :

Find $\mathcal{A}=\left(\mathcal{A}_{1}, \ldots, \mathcal{A}_{n}\right)$, a family of $n$ disjoint subsets of $F^{n}$, such that any of their transversals (see definition 2) will always constitute a set of linearly independent vectors.

Clearly, a family $\mathcal{A}$ of sets solution of the SELIT problem satisfy the completeness property.

The SELIT problem is loosely related to the well known index coding problem [7]: given a server with a wireless broadcast link to several clients that already have some content, what is the minimum number of transmissions necessary to send the information, they want? And with which coding scheme? The index coding literature spawned results such as an equivalence with network coding [8], and capacity region results in the distributed case [9]. SELIT is simpler because it decouples the "capacity" aspect present in the index coding problem; we are unaware of existing constructions of the literature that would provide SELIT solutions ${ }^{3}$.

\section{ON MILIC AND ON SOLUTIONS OF SELIT}

One example of SELIT solution for $n=2$ is in Ex. 1, where $\mathcal{A}_{1}=\left\{\left(\begin{array}{ll}1 & 2\end{array}\right),\left(\begin{array}{ll}2 & 1\end{array}\right)\right\}$, meaning that replies to Interest $I_{1}$ are $Q_{1}=P_{1}+2 P_{2}$ and $Q_{1}^{\prime}=2 P_{1}+P_{2}$; and any of these is linearly independent of any of the two possible replies for $I_{2}$ which are $Q_{2}=P_{1}$ and $Q_{2}^{\prime}=P_{1}+P_{2}$.

\section{Example 1.}

$F=\mathrm{GF}(3), n=2, k=2, \mathcal{A}=\left(\mathcal{A}_{1}, \mathcal{A}_{2}\right)$ with

$\mathcal{A}_{1}=\left\{\left(\begin{array}{ll}1 & 2\end{array}\right),\left(\begin{array}{ll}2 & 1\end{array}\right)\right\}$

$\mathcal{A}_{2}=\left\{\left(\begin{array}{ll}1 & 0\end{array}\right),\left(\begin{array}{ll}1 & 1\end{array}\right)\right\}$

There are exactly 4 possible transversals:

- $T_{1}=\left\{\left(\begin{array}{ll}1 & 2\end{array}\right),\left(\begin{array}{ll}1 & 0\end{array}\right)\right\} \cdot \operatorname{det}\left(\begin{array}{ll}1 & 2 \\ 1 & 0\end{array}\right)=1 \neq 0$

- $T_{2}=\left\{\left(\begin{array}{ll}1 & 2\end{array}\right),\left(\begin{array}{ll}1 & 1\end{array}\right)\right\} .\left|\begin{array}{ll}1 & 2 \\ 1 & 1\end{array}\right|=2 \neq 0$

- $T_{1}=\left\{\left(\begin{array}{ll}2 & 1\end{array}\right),\left(\begin{array}{ll}1 & 0\end{array}\right)\right\} .\left|\begin{array}{ll}2 & 1 \\ 1 & 0\end{array}\right|=1 \neq 0$

- $T_{2}=\left\{\left(\begin{array}{ll}2 & 1\end{array}\right),\left(\begin{array}{ll}1 & 1\end{array}\right)\right\} \cdot\left|\begin{array}{ll}2 & 1 \\ 1 & 1\end{array}\right|=1 \neq 0$

Hence all 4 transversals are sets of 2 linearly independent vectors, and thus $\mathcal{A}$ is a SELIT solution.

\footnotetext{
${ }^{1}$ notice that high diversity is correlated with high cardinality of sets $\mathcal{A}_{i}$ in any case, so after finding large $\mathcal{A}_{i}$, one could check a posteriori their diversity

${ }^{2}$ The problem can also be generalized as $k$-SELIT for finding $k<n$ sets instead of $n$ (with some uses for ICN): this is not explored in this article.

${ }^{3}$ but note that linear codes might map a SELIT to a k-SELIT solution.
} 
Definition 3 (Multiple Interests for Linearly Independent Contents). MILIC is a construction with sets of encoding vectors $\left(\mathcal{A}_{i}\right)_{i \in \llbracket n \rrbracket}$ introduced in [5] as follows: $\forall i \in \llbracket n \rrbracket$,

$$
\mathcal{A}_{i}=\left\{\left(v_{1}, \ldots, v_{n}\right) \in F^{n} \mid v_{i} \neq 0 \text { and } \forall j \in \llbracket i-1 \rrbracket, v_{j}=0\right\} .
$$

Example 2 (MILIC for $n=3$ ).

- $\mathcal{A}_{1}=\left\{\left(\begin{array}{lll}a_{1} & a_{2} & a_{3}\end{array}\right) \mid a_{1} \in F^{*}, a_{2} \in F, a_{3} \in F\right\}$

- $\mathcal{A}_{2}=\left\{\left(\begin{array}{lll}0 & b_{2} & b_{3}\end{array}\right) \mid b_{2} \in F^{*}, b_{3} \in F\right\}$

- $\mathcal{A}_{3}=\left\{\left(\begin{array}{lll}0 & 0 & c_{3}\end{array}\right) \mid c_{3} \in F^{*}\right\}$

Ex. 2 illustrates the MILIC construction [5] when $n=k=$ 3. MILIC is one solution to the SELIT problem.

By construction, any transversal of the MILIC sets is linearly independent. Results in this article originate from attempts to generalize MILIC constructions with matrices where all coefficients are freely picked from predefined sets, with additional constraints, see Def. 6. They are for any field $F$ with $|F|>2$, that is, all excepted $G F(2)$. Theorem 4 states that a solution in this form is a MILIC solution up to a permutation of indices.

\section{InVESTIGATED FAMILIES OF CONSTRUCTIONS}

In this article, we investigate solutions in a form similar to MILIC: this section specifies what "similar" means, after introducing some additional definitions.

We first notice that if $\mathcal{A}=\left(\mathcal{A}_{1}, \ldots, \mathcal{A}_{n}\right)$ is a SELIT solution, one can take one arbitrary vector in each set $v_{i} \in \mathcal{A}_{i}$, and use the set of vectors $\left(v_{i}\right)_{i}$ as a new basis for coordinates. Accordingly, we introduce the concept of canonical family:

Definition 4 (Canonical family of sets).

Let $\mathcal{A}=\left(\mathcal{A}_{1}, \ldots, \mathcal{A}_{n}\right)$ be a family of $n$ subsets of $F^{n}$ :

A canonical family of sets is such that each canonical vector $e_{i}$ is such that $e_{i} \in \mathcal{A}_{i}$ for all $i \in \llbracket n \rrbracket$.

Definition 5 (Canonical and component-wise family of sets). A family $\mathcal{A}=\left(\mathcal{A}_{1}, \ldots, \mathcal{A}_{n}\right)$ of $n$ subsets of $F^{n}$ is a canonical and component-wise family of sets if subset $\mathcal{A}_{i}, \in \llbracket n \rrbracket$ is the union of $e_{i}$ with the $n$-fold cartesian product of sets $A_{i, j}$, where $A_{i, j} \subseteq F$ are parameters of the construction. Such that $\forall i \in \llbracket n \rrbracket$,

$$
\mathcal{A}_{i}=\left\{\left(\begin{array}{lll}
v_{1} & \cdots & v_{n}
\end{array}\right) \mid v_{j} \in A_{i, j} \forall j=1 \ldots n\right\} \cup\left\{e_{i}\right\} .
$$

A canonical and component-wise family of sets $\mathcal{A}$ is fully specified from the table $\mathcal{C}(\mathcal{A})$ of subsets $A_{i, j}$ denoted as

$$
\mathcal{C}(\mathcal{A}) \stackrel{\text { def }}{=}\left\{\begin{array}{ccc}
A_{1,1} & \cdots & A_{1, n} \\
\vdots & \ddots & \vdots \\
A_{n, 1} & \cdots & A_{n, n}
\end{array}\right\}
$$

Example 3. The MILIC construction $\mathcal{A}^{\text {MILIC }}$ in Def. 3 is a canonical and component-wise family, associated with

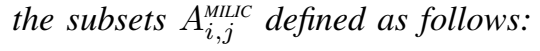

$$
\begin{cases}A_{i, i}^{\text {MILIC }}=F^{*} & \text { if } i=j \\ A_{i, j}^{\text {MILIC }}=\{0\} & \text { if } i<j \\ A_{i, j}^{\text {MILIC }}=F & \text { if } i>j\end{cases}
$$

and we have

$$
\mathcal{C}\left(\mathcal{A}^{\text {MILIC }}\right)=\left\{\begin{array}{cccc}
F^{*} & F & \cdots & F \\
\{0\} & F^{*} & \cdots & F \\
\vdots & \vdots & \ddots & F \\
\{0\} & \{0\} & \cdots & F^{*}
\end{array}\right\}
$$

Notice that the Cartesian products defined in MILIC already contain the canonical unit vectors $e_{i}$.

Example 4. Consider the MILIC construction $\mathcal{A}=$ $\left(\mathcal{A}_{1}, \mathcal{A}_{2}, \mathcal{A}_{3}\right)$ with $n=3$ with $F=G F(3) ; F$ can then be represented as $F=\{0,1,2\}$, and $F^{*}=\{1,2\}$. Then

$$
\mathcal{C}(\mathcal{A})=\left\{\begin{array}{ccc}
\{1,2\} & \{0,1,2\} & \{0,1,2\} \\
\{0\} & \{1,2\} & \{0,1,2\} \\
\{0\} & \{0\} & \{1,2\}
\end{array}\right\}
$$

Definition 6 (Canonical, Diagonal and Restricted Diagonal family of sets $)$. Let $\mathcal{A}=\left(\mathcal{A}_{1}, \mathcal{A}_{2}, \ldots, \mathcal{A}_{n}\right)$ be a canonical and component-wise family of $n$ sets of $F$. We say that it is a diagonal family if there exists $A_{i, j} \subset F, \forall i \in \llbracket n \rrbracket$, $\forall j \in \llbracket n \rrbracket \backslash\{i\}$ such that

$$
\mathcal{C}(\mathcal{A})=\left\{\begin{array}{cccc}
F^{*} & A_{1,2} & \cdots & A_{1, n} \\
A_{2,1} & F^{*} & \cdots & A_{2, n} \\
\vdots & \vdots & \ddots & \vdots \\
A_{n, 1} & A_{n, 2} & \cdots & F^{*}
\end{array}\right\}_{n \times n}
$$

It is a canonical and restricted diagonal family if the offdiagonal sets $\left(A_{i, j}\right)$ additionally satisfy:

$$
\forall i \in \llbracket n \rrbracket, \forall j \in \llbracket n \rrbracket \backslash\{i\}: A_{i, j} \subset F^{*} \text { or } A_{i, j}=\{0\} .
$$

\section{Vi. TRuncated Permutations}

One important property of a canonical component-wise SELIT solution $\mathcal{A}$ for a given $n$ is that one can extract smaller SELIT solutions $\mathcal{A}^{\prime}$ for $n^{\prime}<n$ by removing some sets and some coefficients of vectors of the sets. This is proven at the end of this section in Theorem 1 and will be used as the basis of the proof by induction of our main result in Sect. VII.

We first formally define what is meant by extract, through the definition of a truncated permutation. 
A truncated permutation consists in applying some permutation $\ell_{i}$ to the elements of a vector, then truncating it to the first $k$ elements. It is defined by $\ell_{i} \in \llbracket n \rrbracket$ for $\forall i \in \llbracket k \rrbracket$, such that $\forall j \in \llbracket k \rrbracket: \ell_{i} \neq \ell_{j}$ when $i \neq j$. The indices of the coefficients are specified by a tuple of $k$ different indices $L=\left(\ell_{1}, \ell_{2}, \cdots, \ell_{k}\right)$. Later we use mapping for truncated permutation.

Definition 7 (Mapping of a vector).

Let $v \in F^{n}$ be a vector $\left(\begin{array}{llll}v_{1} & v_{2} & \cdots & v_{n}\end{array}\right)$ and consider a tuple of $k$ different indices $\left(\ell_{1}, \ell_{2}, \cdots, \ell_{k}\right)$. The mapping of $v$ is

$$
T P\left(v, \ell_{1}, \ell_{2}, \ldots, \ell_{k}\right) \stackrel{\text { def }}{=}\left(\begin{array}{llll}
v_{\ell_{1}} & v_{\ell_{2}} & \cdots & v_{\ell_{k}}
\end{array}\right) \in F^{k}
$$

This definition can be extended to a family of subsets in a way that their diagonal structure is preserved.

Definition 8 (Mapping of a family of sets). Let $\mathcal{A}=\left(\mathcal{A}_{1}, \ldots, \mathcal{A}_{n}\right)$ be a family of sets of $F^{n}$.

The mapping of $\mathcal{A}$, denoted $\operatorname{TP}\left(\mathcal{A}, \ell_{1}, \ell_{2}, \ldots, \ell_{k}\right)$ with the indices $\left(\ell_{1}, \ell_{2}, \ldots, \ell_{k}\right)$ is the family of sets $\mathcal{A}^{\prime}$ with:

$$
\begin{aligned}
& \mathcal{A}^{\prime}=\left(\mathcal{A}_{1}^{\prime}, \ldots, \mathcal{A}_{k}^{\prime}\right) \\
& \mathcal{A}_{i}^{\prime}=\left\{T P\left(v, \ell_{1}, \ell_{2}, \ldots, \ell_{k}\right) \mid \forall v \in \mathcal{A}_{\ell_{i}}\right\}, \forall i \in \llbracket k \rrbracket
\end{aligned}
$$

When $\mathcal{A}$ is a component-wise family with coefficients from the sets $\left(A_{i j}\right)_{i \in \llbracket n \rrbracket, j \in \llbracket n \rrbracket}$, and we define a notation for the table of coefficients of the mapping:

$$
\begin{array}{r}
\mathcal{P}\left(\mathcal{A}, \ell_{1}, \ell_{2}, \ldots, \ell_{k}\right) \stackrel{\text { def }}{=} \mathcal{C}\left(T P\left(\mathcal{A}, \ell_{1}, \ell_{2}, \ldots, \ell_{k}\right)\right) \\
\mathcal{P}\left(\mathcal{A}, \ell_{1}, \ell_{2}, \ldots, \ell_{k}\right)=\left\{\begin{array}{cccc}
A_{\ell_{1} \ell_{1}} & A_{\ell_{1} \ell_{2}} & \cdots & A_{\ell_{1} \ell_{k}} \\
A_{\ell_{2} \ell_{1}} & A_{\ell_{2} \ell_{2}} & \cdots & A_{\ell_{2} \ell_{k}} \\
\vdots & \vdots & \ddots & \vdots \\
A_{\ell_{k} \ell_{1}} & A_{\ell_{n} \ell_{2}} & \cdots & A_{\ell_{k} \ell_{k}}
\end{array}\right\}
\end{array}
$$

Example 5. For MILIC with $n=3$ (defined in example 4), we have the following examples of mapping:

$$
\begin{aligned}
& \mathcal{P}(\mathcal{A}, 1,2,3)=\left\{\begin{array}{ccc}
F^{*} & F & F \\
\{0\} & F^{*} & F \\
\{0\} & \{0\} & F^{*}
\end{array}\right\} \text { with } \begin{array}{l}
F=\{0,1,2\} \\
F^{*}=\{1,2\}
\end{array} \\
& \mathcal{P}(\mathcal{A}, 1,2)=\left\{\begin{array}{ll}
F^{*} & F \\
\{0\} & F^{*}
\end{array}\right\} \text { and } \mathcal{P}(\mathcal{A}, 2,1)=\left\{\begin{array}{cc}
F^{*} & \{0\} \\
F & F^{*}
\end{array}\right\} .
\end{aligned}
$$

Example 6. When $\mathcal{A}=\left(\mathcal{A}_{1}, \mathcal{A}_{2}, \ldots, \mathcal{A}_{n}\right)$ is a MILIC construction then for any set of $k$ indices $\ell_{1}, \ell_{2}, \ldots, \ell_{k}$ with $\ell_{1}<\ell_{2} \ldots<\ell_{k}$, we have:

$$
\mathcal{P}\left(\mathcal{A}, \ell_{1}, \ell_{2}, \ldots, \ell_{k}\right)=\left\{\begin{array}{ccccc}
F^{*} & F & F & \cdots & F \\
\{0\} & F^{*} & F & \cdots & F \\
\{0\} & \{0\} & F^{*} & \cdots & F \\
\vdots & \vdots & \vdots & \ddots & \vdots \\
\{0\} & \{0\} & \{0\} & \cdots & F^{*}
\end{array}\right\}
$$

Theorem 1 (Mapping Theorem for Canonical Solutions). Let $\mathcal{A}$ be a canonical family of sets, that is a SELIT solution to the SELIT problem for $n$, and $\ell_{1}, \ell_{2}, \ldots, \ell_{k}$ be a sequence of distinct $k$ indices in [[n]].

Then $\mathcal{A}^{\prime}=\operatorname{TP}\left(\mathcal{A}, \ell_{1}, \ell_{2}, \ldots, \ell_{k}\right)$ is a family of $k$ sets of $F^{k}$ and a SELIT solution to the SELIT problem for dimension $k$.

Proof. By contradiction: assume $\mathcal{A}^{\prime}$ is not a SELIT solution. It implies that there exist $w_{i} \in \mathcal{A}_{i}^{\prime}, \forall i \in \llbracket k \rrbracket$ and $\alpha \in F^{k}$, $\alpha \neq 0$ such that $\alpha_{1} w_{1}+\alpha_{2} w_{2}+\ldots+\alpha_{k} w_{k}=0$. $\mathcal{A}^{\prime}$ is a family of subsets that are mapping of $\mathcal{A}$, which implies that each vector $w_{i} \in \mathcal{A}_{i}^{\prime}$ is a mapping of some vector $v_{i} \in \mathcal{A}_{i}$ from (7). Then

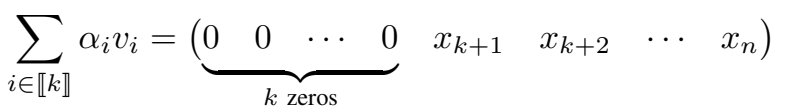

up to a permutation of the entries. Since $\mathcal{A}$ is a canonical family of sets, each $\mathcal{A}_{j}$ includes the canonical vector $e_{j}$, and then

$$
\sum_{\forall i \in \llbracket k \rrbracket} \alpha_{i} v_{i}-\sum_{\forall i \in\{k+1, \ldots, n\}} x_{i} e_{i}=0
$$

which is a contradiction with the fact that $\mathcal{A}$ is a SELIT solution. Hence $\mathcal{A}^{\prime}$ must be a SELIT solution.

\section{PROPERTIES OF CANONICAL AND RESTRICTED Diagonal SOLUTIONS OF THE SELIT PROBLEM}

In this entire section, we consider one solution of the SELIT problem $\mathcal{A}$ that is canonical, component-wise, and restricted diagonal. Recall that by the previous definitions we can write:

$$
\left\{\begin{array}{l}
\mathcal{A}=\left(\mathcal{A}_{1}, \ldots, \mathcal{A}_{n}\right), \text { with } \forall i \in \llbracket n \rrbracket: e_{i} \in \mathcal{A}_{i} \\
\mathcal{C}(\mathcal{A})=\left\{\begin{array}{cccc}
F^{*} & A_{1,2} & \cdots & A_{1, n} \\
A_{2,1} & F^{*} & \ddots & \vdots \\
\vdots & \ddots & \ddots & A_{n-1, n} \\
A_{n, 1} & \ldots & A_{n, n-1} & F^{*}
\end{array}\right\} \\
\text { with } \forall i \in \llbracket n \rrbracket, \forall j \in \llbracket n \rrbracket \backslash\{i\}: A_{i, j} \subset F^{*} \text { or } A_{i, j}=\{0\}
\end{array}\right.
$$

and we will use these definitions.

Let $\ell_{1}, \ell_{2}$ be two different integers in $\llbracket n \rrbracket$. The $2 \times 2$ mapping, $\mathcal{P}\left(\mathcal{A}, \ell_{1}, \ell_{2}\right)=\left\{\begin{array}{cc}F^{*} & A_{\ell_{1}, \ell_{2}} \\ A_{\ell_{2}, \ell_{1}} & F^{*}\end{array}\right\}$ involves only $A_{\ell_{1}, \ell_{2}}$ and $A_{\ell_{2}, \ell_{1}}$, that have an important property:

Lemma 1 (Non-Zero Set Exclusion). Consider $n>1$. Let $\mathcal{A}$ be a SELIT solution as in (8). Let $\ell_{1}, \ell_{2}$ be two different integers in $\llbracket n \rrbracket$.

Then $A_{\ell_{1}, \ell_{2}}=\{0\}$ or $A_{\ell_{2}, \ell_{1}}=\{0\}$ or both are $\{0\}$

Proof. By contradiction: assume that both $A_{\ell_{1}, \ell_{2}} \neq\{0\}$ and $A_{\ell_{2}, \ell_{1}} \neq\{0\}$. Then from (8), $A_{\ell_{1}, \ell_{2}} \subset F^{*}$, and $A_{\ell_{2}, \ell_{1}} \subset F^{*}$, hence $0 \notin A_{\ell_{1}, \ell_{2}}$ and $0 \notin A_{\ell_{2}, \ell_{1}}$. Let $\mathcal{A}^{\prime}=\left(\mathcal{A}_{1}^{\prime}, \ldots, \mathcal{A}_{2}^{\prime}\right)$ be the mapping of $\mathcal{A}$, i.e. $\mathcal{A}^{\prime}=\mathcal{P}\left(\mathcal{A}, \ell_{1}, \ell_{2}\right)$. We know that: $\mathcal{C}\left(\mathcal{A}^{\prime}\right)=\mathcal{P}\left(\mathcal{A}, \ell_{1}, \ell_{2}\right)=\left\{\begin{array}{cc}F^{*} & A_{\ell_{1}, \ell_{2}} \\ A_{\ell_{2}, \ell_{1}} & F^{*}\end{array}\right\}$ 
Take arbitrary values $a_{1} \in \mathcal{A}_{\ell_{1}, \ell_{2}}$, and $a_{2} \in \mathcal{A}_{\ell_{2}, \ell_{1}}$, necessarily non-zero, and let $v=\left(\begin{array}{ll}a_{2} & a_{1}\end{array}\right)$. Then $v \in \mathcal{A}_{1}^{\prime}$ and $v \in \mathcal{A}_{2}^{\prime}$, and $v$ is linearly dependent with itself.

Thus $\mathcal{A}^{\prime}$ is not a SELIT solution and by theorem 1, neither is $\mathcal{A}$. This is a contradiction, therefore the initial assumption that both $A_{\ell_{1}, \ell_{2}}$ and $A_{\ell_{2}, \ell_{1}}$ are not $\{0\}$ must be false, hence the lemma.

This lemma allows us to further characterize SELIT solutions in binary relations.

Definition 9 (Binary Relations from $\mathcal{A}$ ). Consider $\mathcal{A}$ as in (8), $\ell_{1} \in \llbracket n \rrbracket$ and $\ell_{2} \in \llbracket n \rrbracket$ with $\ell_{1} \neq \ell_{2}$. We can introduce the binary relations on $\ell_{1}$ and $\ell_{2}$, depending on whether $A_{\ell_{1}, \ell_{2}}$ and $A_{\ell_{2}, \ell_{1}}$ are $\{0\} \quad$ (we also indicate how $P \stackrel{\text { def }}{=} \mathcal{P}\left(\mathcal{A}, \ell_{1}, \ell_{2}\right)$ looks like):

$$
\begin{aligned}
& \ell_{1} \stackrel{\mathcal{A}}{\prec} \ell_{2} \text { if only } A_{\ell_{2}, \ell_{1}}=\{0\} ; \text { thus: } P=\left\{\begin{array}{cc}
F^{*} & A_{\ell_{1}, \ell_{2}} \\
\{0\} & F^{*}
\end{array}\right\} \\
& \ell_{2} \stackrel{\mathcal{A}}{\prec} \ell_{1} \text { if only } A_{\ell_{1}, \ell_{2}}=\{0\} ; \text { thus: } P=\left\{\begin{array}{cc}
F^{*} & \{0\} \\
A_{\ell_{2}, \ell_{1}} & F^{*}
\end{array}\right\} \\
& \ell_{2} \stackrel{\mathcal{A}}{\sim} \ell_{1} \text { else when both are }\{0\} ; \text { thus: } P=\left\{\begin{array}{ll}
F^{*} & \{0\} \\
\{0\} & F^{*}
\end{array}\right\}
\end{aligned}
$$

$$
\text { Observe that: } \ell_{1} \stackrel{A}{\gtrless} \ell_{2} \Longleftrightarrow A_{\ell_{1}, \ell_{2}}=\{0\}
$$

The overscript $\mathcal{A}$ makes clear that the binary relations depend on $\mathcal{A}$. To simplify, when there is no ambiguity from the context, we will write $\prec$ instead of $\stackrel{\mathcal{A}}{\prec}$.

We now start with a lemma on the determinant of matrices.

Lemma 2. Consider the sets $B_{i, j}, j \in \llbracket k \rrbracket, i \in \llbracket j-1 \rrbracket$, the sets $D_{i} \subset F^{*}, i \in \llbracket k \rrbracket \subset F$, and the set set of matrices $\mathcal{M}_{k}$ defined as

$$
\begin{aligned}
& \mathcal{M}_{k}=\left\{\left(\begin{array}{cccccc}
d_{1} & b_{1,2} & b_{1,3} & \cdots & b_{1, k-1} & b_{1, k} \\
c_{1} & d_{2} & b_{2,3} & \cdots & b_{2, k-1} & b_{2, k} \\
0 & c_{2} & d_{3} & \cdots & b_{3, k-1} & b_{3, k} \\
\vdots & \vdots & \ddots & \ddots & \vdots & \vdots \\
0 & 0 & 0 & \ddots & d_{k-1} & b_{k-1, k} \\
0 & 0 & 0 & \cdots & c_{k-1} & d_{k}
\end{array}\right)\right. \\
& \mid b_{i, j} \in B_{i, j}, \forall j \in \llbracket k \rrbracket, \forall i \in \llbracket j-1 \rrbracket,
\end{aligned}
$$

and $d_{i} \in D_{i}, \forall i \in \llbracket k \rrbracket$, and $\left.c_{i} \in F^{*}, \forall i \in \llbracket k-1 \rrbracket\right\}$,

Then there exists a matrix $M \in \mathcal{M}_{k}$ such that $\operatorname{det}(M) \neq$ 0

Proof. For $k=2$ : consider a $2 \times 2$ matrix $M_{2} \in \mathcal{M}$

$$
M_{2}=\left(\begin{array}{cc}
d_{1} & b_{1,2} \\
c_{1} & d_{2}
\end{array}\right)
$$

where $d_{1}, d_{2}$, arbitrarily chosen from the predefined sets $D_{i} \subset F^{*}, i=1,2$, are necessarily non-zero; $b_{1,2} \in B_{1,2}$ is also arbitrary chosen, and might be zero. If $b_{1,2}=0$ then $\operatorname{det}\left(M_{2}\right) \neq 0$. Otherwise if $b_{1,2} \neq 0$ then for each value of $c_{1}$ the $\operatorname{det}\left(M_{2}\right)$ will have a different value, thus there exists $c_{1}$ with $\operatorname{det}\left(M_{2}\right) \neq 0$. Thus the result for $k=2$.

We prove the general case by induction. Assume the property is proven for $\mathcal{M}_{k-1}$ for some $k>1$. The determinant of all $k \times k$ matrices $M_{k} \in \mathcal{M}_{k}$ can be written as

$$
\operatorname{det}\left(M_{k}\right)=d_{1} \operatorname{det}\left(M_{k-1}^{\prime}\right)-c_{1} \operatorname{det}(Q)
$$

where $M_{k-1}^{\prime} \in \mathcal{M}_{k-1}$ and $Q$ is some $(k-1) \times(k-1)$ matrix. Neither $M_{k-1}^{\prime}$ nor $Q$ involves the coefficient $c_{1}$. From the induction assumption, we know that there exists $M_{k-1}^{\prime} \in \mathcal{M}_{k-1}$ such that $\operatorname{det}\left(M_{k-1}^{\prime}\right) \neq 0$. The matrix $M_{k}$ is built from $M_{k-1}^{\prime}$, selecting arbitrarily the remaining coefficients from their possible sets, except $c_{1}$. If $\operatorname{det}(Q)=0$, then $\forall c_{1} \in F^{*}, \operatorname{det}\left(M_{k}\right) \neq 0$. If $\operatorname{det}(Q) \neq 0$, $\operatorname{det}\left(M_{k}\right)$ will take as many different values as $c_{1}$ and $\exists c_{1} \in F^{*}$ such that $\operatorname{det}\left(M_{k}\right) \neq 0$. Assuming the property the lemma true for $\mathcal{M}_{k-1}$, it is thus also true for $\mathcal{M}_{k}$. Since it is true for $\mathcal{M}_{2}$, the lemma is proven.

Theorem 2. Let $\mathcal{A}$ be a SELIT solution as in (8). Let $k>1$. Consider a sequence of $k$ indices $L=\left\{\ell_{1}, \ell_{2}, \ldots, \ell_{k}\right\}$, such that $\ell_{1} \prec \ell_{2}, \ell_{2} \prec$ $\ell_{3}, \ldots$, and $\ell_{k-1} \prec \ell_{k}$, then $\ell_{k} \nprec \ell_{1}$.

Proof. By the property of $\prec$ in (9) and (10), $\ell_{1} \prec \ell_{2}$ and $\ell_{2} \prec \ell_{1}$ exclude each other, and the theorem is proven for $k=2$. We will prove the theorem for $k>2$, by induction, assuming that it was proven for $2,3, \ldots, k-1$.

Consider a sequence of $k$ indices $\mathrm{L}=\left\{\ell_{1}, \ell_{2}, \ldots, \ell_{k}\right\}$, that satisfies $\ell_{1} \prec \ell_{2}, \ell_{2} \prec \ell_{3}, \ldots$, and $\ell_{k-1} \prec \ell_{k}$.

For any $i \in \llbracket k \rrbracket$ and $j \in \llbracket k \rrbracket$ with $i<j$ and $(i, j) \neq(1, k)$, we can apply the induction hypothesis for $\ell_{i} \prec \ell_{i+1}, \ell_{i+1} \prec$ $\ell_{i+2}, \ldots$, and $\ell_{j-1} \prec \ell_{j}$, which forms a chain of $k^{\prime}=j-i+$ $1 \leq k-1$ binary relations. Thus $\ell_{j} \nprec \ell_{i}$ and from (12),

$\forall i \in \llbracket k \rrbracket, \forall j \in \llbracket k \rrbracket$ with $i<j,(i, j) \neq(1, k): \mathcal{A}_{\ell_{j}, \ell_{i}}=\{0\}$

Notice that these are all the elements in the lower triangle of the table $\mathcal{P}\left(\mathcal{A}, \ell_{1}, \ell_{2}, \ldots, \ell_{k}\right)$, and are $\{0\}$, except for the diagonal and for $A_{\ell_{k}, \ell_{1}}$.

$$
P_{\mathrm{L}}=\left\{\begin{array}{ccccc}
F^{*} & A_{\ell_{1}, \ell_{2}} & \cdots & A_{\ell_{1}, \ell_{k-1}} & A_{\ell_{1}, \ell_{k}} \\
\{0\} & F^{*} & \cdots & A_{\ell_{2}, \ell_{k-1}} & A_{\ell_{2}, \ell_{k}} \\
\vdots & \ddots & \ddots & \vdots & \vdots \\
\{0\} & \{0\} & \ddots & F^{*} & A_{\ell_{k-1}, \ell_{k}} \\
A_{\ell_{k}, \ell_{1}} & \{0\} & \cdots & \{0\} & F^{*}
\end{array}\right\} .
$$

Consider $\mathcal{A}^{\prime}=\operatorname{TP}\left(\mathcal{A}, \ell_{1}, \ell_{2}, \ldots, \ell_{k}\right)$ a mapping of the SELIT solution $\mathcal{A}$. Consider the set of matrices $\mathcal{Q}_{\mathcal{A}^{\prime}}$ constructed from vectors obtained by picking one vector in each set of $\mathcal{A}^{\prime}$. Each matrix $M=\left(m_{i, j}\right)$ in $\mathcal{Q}_{\mathcal{A}^{\prime}}$ is obtained by picking a coefficient in the table $P_{\mathrm{L}}$ in (13) at the corresponding position. Its determinant can be written as:

$$
\operatorname{det}(M)=m_{1,1} \operatorname{det}(G)-m_{k, 1} \operatorname{det}(H)
$$


where $m_{1,1} \in F^{*}, G$ is a triangular matrix (with diagonal elements in $\left.F^{*}\right), m_{k, 1} \in A_{\ell_{k}, \ell_{1}}$ and $H$ is a matrix in form of $\mathcal{M}_{k-1}$ from Lemma 2. This implies that coefficients present in matrix $H$ can be selected such that $\operatorname{det}(H) \neq 0$. Moreover, $\operatorname{det}(G) \neq 0$ as $\operatorname{det}(G)$ is the product of nonzero elements. If $m_{k, 1}$ is not zero, then there exists a value $m_{1,1}=m_{k, 1} \operatorname{det}(H) / \operatorname{det}(G) \in F^{*}$ such that $\operatorname{det}(M)=0$, and then the corresponding vectors of $M$ would be linearly dependent, which contradicts the fact that $\mathcal{A}^{\prime}$ (thus $\mathcal{A}$ ) is a SELIT solution. Thus $m_{k, 1}$ must always be 0 , hence, $0 \in A_{\ell_{k}, \ell_{1}}$, and from (8), this implies that $A_{\ell_{k}, \ell_{1}}=\{0\}$.

From (12), this now implies that $\ell_{k} \nprec \ell_{1}$ and this concludes the proof by induction, hence the theorem.

Theorem 3. Any canonical and restricted diagonal family $\mathcal{A}=\left(\mathcal{A}_{1}, \ldots, \mathcal{A}_{n}\right)$ that is a solution of SELIT, is included in a family $\mathcal{B}=\left(\mathcal{B}_{1}, \ldots, \mathcal{B}_{n}\right)$ which is a permutation of lines and rows of the MILIC construction for $n$ (where "included" means that $\forall i \in \llbracket n \rrbracket, \mathcal{A}_{i} \subset \mathcal{B}_{i}$ )

Proof. The binary relation $\prec$ is acyclic as a direct consequence of Theorem 2. We can use classical results to build a total order embedding it, see for instance [10]: let $\triangleleft$ be the transitive closure of $\prec$; the transitive closure of an acyclic relation is irreflexive (see [10]). Now, by the Szpilrajn extension theorem [11], for a transitive and irreflexive relation, there exists a total order, that includes it, denoted $\lll$. We can reorder the indices $\llbracket n \rrbracket$ as a sequence $\mathrm{L}=\left\{\ell_{1}, \ell_{2}, \ldots, \ell_{n}\right\}$ using the total order such that $\ell_{1} \lll \ell_{2} \lll \ldots \ell_{n-1} \lll \ell_{n}$. The mapping of $\mathcal{A}$ with $\mathrm{L}$ (actually a permutation) is given by:

$$
\mathcal{P}\left(\mathcal{A}, \ell_{1}, \ell_{2}, \ldots, \ell_{n}\right)=\left\{\begin{array}{cccc}
F^{*} & A_{\ell_{1}, \ell_{2}} & \cdots & A_{\ell_{1}, \ell_{n}} \\
A_{\ell_{2}, \ell_{1}} & F^{*} & \cdots & A_{\ell_{2}, \ell_{n}} \\
\vdots & \vdots & \ddots & \vdots \\
A_{\ell_{n}, \ell_{1}} & A_{\ell_{n}, \ell_{2}} & \cdots & F^{*}
\end{array}\right\}
$$

Consider any $\ell_{i} \in \llbracket n \rrbracket, \ell_{j} \in \llbracket n \rrbracket$ and $\ell_{i} \neq \ell_{j}$. Assume that $A_{\ell_{i}, \ell_{j}} \neq\{0\}$. From (12), $A_{\ell_{i}, \ell_{j}} \neq\{0\}$ iff $\ell_{i} \prec \ell_{j}$. Then $\ell_{i} \prec \ell_{j}$ implies that $\ell_{i} \triangleleft \ell_{j}$ and consequently $\ell_{i} \lll \ell_{j}$. And then $i<j$ (because $\ell_{i} \lll \ell_{j} \Longleftrightarrow i<j$ ), and this element $A_{\ell_{i}, \ell_{j}}$ must be in the upper triangle.

As a consequence, if $\ell_{i}, \ell_{j}$, correspond to the lower triangle (e.g. $j<i$ ) then $A_{\ell_{i}, \ell_{j}}=\{0\}$. This proves that the family $\mathcal{A}^{\prime}=\mathcal{P}(\mathcal{A}, \mathrm{L})$ has a triangular matrix, hence is included in the MILIC construction for $n$, shown in (7). $\mathcal{A}^{\prime}$ is obtained through a permutation of $\mathcal{A}$, hence the theorem, with $\mathcal{B}$ obtained through the inverse permutation of the MILIC construction for $n$.

Now the most general form of the theorem is obtained by no longer considering "restricted diagonal" families, but any "diagonal" families:
Theorem 4. Any canonical and diagonal family $\mathcal{A}=$ $\left(\mathcal{A}_{1}, \ldots, \mathcal{A}_{n}\right)$ that is a solution of SELIT, is included in a family $\mathcal{B}=\left(\mathcal{B}_{1}, \ldots, \mathcal{B}_{n}\right)$ which is a permutation of lines and rows of the MILIC construction for $n$ (where "included" means that $\left.\forall i \in \llbracket n \rrbracket, \mathcal{A}_{i} \subset \mathcal{B}_{i}\right)$.

Proof. Let $\left(A_{i, j}\right)$ be the sets of coefficients in $\mathcal{C}(A)$. Let $\mathcal{A}^{\prime}$ be the family defined from its sets of coefficients $\left(A_{i, j}^{\prime}\right)$ from $\mathcal{C}\left(A^{\prime}\right)$ selected as follows:

$\forall i \in \llbracket n \rrbracket, \forall j \in \llbracket n \rrbracket:$ if $A_{i, j} \neq\{0\}$ then $A_{i, j}^{\prime} \stackrel{\text { def }}{=} A_{i, j} \backslash\{0\}$ otherwise $A_{i, j}^{\prime} \stackrel{\text { def }}{=}\{0\}$.

$\mathcal{A}^{\prime}$ is now a restricted diagonal solution, hence Theorem 3 can be applied, and $\mathcal{B}$, a permutation of a MILIC construction can be found such that $\mathcal{A}^{\prime}$ is included in $\mathcal{B}$. Now the only difference between $\mathcal{A}^{\prime}$ and $\mathcal{A}$, is that vectors of transversals of $\mathcal{A}$ may have 0 in coefficient positions where vectors of transversals of $\mathcal{A}^{\prime}$ might not: but then they would still be included in $\mathcal{B}$, hence $\mathcal{A}$ is included in $\mathcal{B}$, hence the theorem.

\section{Alternate Algebraic SELIT solutions}

The details of the proofs in this article gave us insights on the reasons why our constructions have specific structures. Consider $M=\left(\begin{array}{ll}x & a \\ b & c\end{array}\right)$. We can write $\operatorname{det}(M)=0 \Longleftrightarrow$ $x=a b c^{-1}$. When $x$ can be selected freely from $F^{*}$, we can make $\operatorname{det}(M)=0$, unless $a, b$ or $c$ must be zero. Notice alternately that if $a, b$, and/or $c$ can take several values, the generalizations of the Cauchy-Davenport theorem show that $a b c^{-1}$ will take even more values, hence making $\operatorname{det}(M)=0$ becomes easier, unless, for instance, they are in a subgroup.

This is the insight that leads us to alternate families of SELIT solutions:

Theorem 5. Let $H_{0}=\{0\}$, and let $H_{1} \subset \ldots \subset H_{n}$ be different subfields of $F$ and denote $\forall i \in \llbracket n \rrbracket, C_{i} \stackrel{\text { def }}{=}$ $H_{i} \backslash H_{i-1}$. Let $\mathcal{A}$ be the "Matryoshka" set family" such that $\mathcal{C}(\mathcal{A})$ is:

$$
\left(\begin{array}{cccccccc}
C_{n} & C_{n-1} & C_{n-1} & \cdots & C_{n-1} & C_{n-1} & C_{n-1} & C_{n-1} \\
C_{n-1} & C_{n-1} & C_{n-2} & \cdots & C_{n-2} & C_{n-2} & C_{n-2} & C_{n-2} \\
C_{n-1} & C_{n-2} & C_{n-2} & \cdots & C_{n-3} & C_{n-3} & C_{n-3} & C_{n-3} \\
\vdots & \vdots & \vdots & & \vdots & \vdots & \vdots & \\
C_{n-1} & C_{n-2} & C_{n-3} & \cdots & C_{4} & C_{3} & C_{3} & C_{3} \\
C_{n-1} & C_{n-2} & C_{n-3} & \cdots & C_{3} & C_{3} & C_{2} & C_{2} \\
C_{n-1} & C_{n-2} & C_{n-3} & \cdots & C_{3} & C_{2} & C_{2} & C_{1} \\
C_{n-1} & C_{n-2} & C_{n-3} & \cdots & C_{3} & C_{2} & C_{1} & C_{1}
\end{array}\right)
$$

Then $\mathcal{A}$ is a SELIT solution.

${ }^{a}$ Note that given a finite field $F$, the "Matryoshka" construction is only possible until a fixed $n$, which depends on existing $F$ subfields. But for any $n$, one can select a finite field $F$ that allows the construction,.

Proof. Pick an arbitrary transversal from $\mathcal{A}$, and write the matrix $M_{n}$ of its vectors. Then one can write: $\operatorname{det}\left(M_{n}\right)=a_{n} \operatorname{det}\left(M_{n-1}\right)+\ldots+(-1)^{n-1} a_{1} \operatorname{det}\left(B_{1}\right)$ where 
$a_{n} \in c_{n}, a_{n-1} \in c_{n-1} \ldots a_{1} \in c_{n-1}$ and $M_{n-1}$ is a matrix with a similar form as $M_{n}$ of size $(n-1) \times(n-1)$ and $B_{1}, \ldots B_{n-1}$ are matrices with coefficients in $H_{n-1}$. $\operatorname{det}\left(M_{n}\right)=a_{n} \operatorname{det}\left(M_{n-1}\right)+h$ where $h \in H_{n-1}$.

If $\operatorname{det}\left(M_{n-1}\right) \in H_{n-1}$ and $\operatorname{det}\left(M_{n-1}\right) \neq 0$, then it is not possible to take $a_{n}=-\operatorname{det}\left(M_{n-1}\right)^{-1} h \in H_{n-1}$ because $a_{n+1} \in c_{n}=H_{n} \backslash H_{n-1}$, hence $\operatorname{det}\left(M_{n}\right) \neq 0$, and still $\operatorname{det}\left(M_{n}\right) \in H_{n}$. This is the basis for a proof of induction that $\operatorname{det}\left(M_{n}\right) \neq 0$, knowing that for $n=1$ the property is true. And hence $\mathcal{A}$ is a SELIT solution.

\section{CONCLUSION}

In this article, we have introduced a new formal problem: the SELIT problem. We have proven that a large class of solutions for $|F| \geq 3$, where one can pick the coefficient vectors from fixed sets and with additional constraints, are essentially a version of the MILIC construction previously introduced. One example of an alternate construction was provided. Open questions and future work include the following: what can be said for families of solutions that are not canonical (which do not include the canonical vectors)? What about $k$-SELIT where the family $\mathcal{A}$ contains only $k<n$ elements? Can other constructions be proposed (using coding theory results)?

\section{REFERENCES}

[1] V. Jacobson, D. K. Smetters, J. D. Thornton, M. F. Plass, N. H. Briggs, and R. L. Braynard. Networking named content. In Proc. ACM CONEXT, pages 1-12. ACM, 2009.

[2] Rudolf Ahlswede, Ning Cai, Shuo-yen Robert Li, and Raymond W. Yeung. Network Information Flow. IEEE Transactions on Information Theory, 46(4):1204-1216, 2000.

[3] M.-J. Montpetit, C. Westphal, and D. Trossen. Network coding meets information-centric networking: An architectural case for information dispersion through native network coding. MobiHoc, pages 31-36, 2012.

[4] Kazuhisa Matsuzono, Hitoshi Asaeda, and Cedric Westphal. Network Coding for Content-Centric Networking / Named Data Networking: Requirements and Challenges. Internet-Draft draft-irtf-nwcrg-nwc-cenreqs-04, IETF, Sep 2020. Work in Progress.

[5] H Malik, C Adjih, C Weidmann, and M Kieffer. MICN: a Network Coding Protocol for ICN with Multiple Distinct Interests per Generation. arXiv preprint arXiv:2007.01128, 2020.

[6] H. Malik, C. Adjih, M. Kieffer, and C. Weidmann. Analysis of the properties of NetcodICN protocols. In CORES 2020, Lyon, France, September 2020.

[7] Y. Birk and T. Kol. Informed-source coding-on-demand (iscod) over broadcast channels. In IEEE INFOCOM, pages 1257-1264, 1998.

[8] M. Effros, S. El Rouayheb, and M. Langberg. An equivalence between network coding and index coding. IEEE Trans. Inf. Theory, 61(5):24782487, 2015.

[9] Y. Liu, P. Sadeghi, F. Arbabjolfaei, and Y. Kim. Capacity theorems for distributed index coding. IEEE Transactions on Information Theory, 66(8):4653-4680, 2020.

[10] Theodore C Bergstrom. Maximal elements of acyclic relations on compact sets. Journal of Economic Theory, 10(3):403 - 404, 1975

[11] Edward Szpilrajn. Sur l'extension de l'ordre partiel. Fundamenta mathematicae, 1(16):386-389, 1930. 